Review Article

\title{
The Relationship between Branched-Chain Amino Acid Related Metabolomic Signature and Insulin Resistance: A Systematic Review
}

\author{
Xue Zhao, ${ }^{1}$ Qing Han, ${ }^{2}$ Yujia Liu, ${ }^{1}$ Chenglin Sun, ${ }^{1}$ Xiaokun Gang, and Guixia Wang ${ }^{1}$ \\ ${ }^{1}$ Department of Endocrinology and Metabolism, The First Hospital of Jilin University, Changchun 130021, China \\ ${ }^{2}$ Hospital of Orthopedics, The Second Hospital of Jilin University, Changchun 130021, China \\ Correspondence should be addressed to Xiaokun Gang; insightful@126.com and Guixia Wang; gwang168@jlu.edu.cn
}

Received 10 April 2016; Revised 16 July 2016; Accepted 1 August 2016

Academic Editor: Konstantinos Kantartzis

Copyright (c) 2016 Xue Zhao et al. This is an open access article distributed under the Creative Commons Attribution License, which permits unrestricted use, distribution, and reproduction in any medium, provided the original work is properly cited.

\begin{abstract}
Recent studies have shown the positive association between increased circulating BCAAs (valine, leucine, and isoleucine) and insulin resistance (IR) in obese or diabetic patients. However, results seem to be controversial in different races, diets, and distinct tissues. Our aims were to evaluate the relationship between BCAA and IR as well as later diabetes risk and explore the phenotypic and genetic factors influencing BCAA level based on available studies. We performed systematic review, searching MEDLINE, EMASE, ClinicalTrials.gov, the Cochrane Library, and Web of Science from inception to March 2016. After selection, 23 studies including 20,091 participants were included. Based on current evidence, we found that BCAA is a useful biomarker for early detection of IR and later diabetic risk. Factors influencing BCAA level can be divided into four parts: race, gender, dietary patterns, and gene variants. These factors might not only contribute to the elevated BCAA level but also show obvious associations with insulin resistance. Genes related to BCAA catabolism might serve as potential targets for the treatment of IR associated metabolic disorders. Moreover, these factors should be controlled properly during study design and data analysis. In the future, more largescale studies with elaborate design addressing BCAA and IR are required.
\end{abstract}

\section{Introduction}

Obesity, one of the escalating global health problems, is a major risk factor for the onset and development of diabetes mellitus, metabolic syndrome, cancer, and other chronic disorders $[1,2]$. Insulin resistance (IR) is known to play a significant role in obesity-related metabolic disturbances $[3,4]$. Several mechanisms are known to be responsible for IR, including carbohydrate metabolism, fat metabolism, and protein metabolism [5]. Despite many years of research, the exact mechanisms between IR and obesity-related metabolic complications are still not fully established. Thus, accurate biomarkers or parameters reflecting insulin resistant state and metabolic risks are essential to understand its mechanism and to prevent obesity-related complications.

Metabolomics is an analytical approach that aims to detect and quantify endogenous small molecule metabolites $(<1,500 \mathrm{Da})[6]$. Recent studies have suggested that altered metabolites or metabolomics profiles could predict specific metabolic diseases with high accuracy and help to understand related fundamental mechanisms as well as affected metabolic pathways [7, 8]. In addition, metabolomics approach is beneficial to classify personalized "metabolic signature" and make it possible to suggest ideal and individualized therapies effectively [9]. Variable kinds of metabolomics techniques have been applied to generate metabolic profiles from blood, urea, or tissues in human, such as ultraperformance liquid chromatography (UPLC), nuclear magnetic resonance spectroscopy (NMR), and tandem mass spectrometry (MS/MS).

BCAAs are essential amino acids, changing along with the consumption of a protein-containing meal, which include valine, leucine, and isoleucine [10]. Previous studies using metabolomics approaches reported that insulin resistant rats and human had an increased level of circulating BCAArelated metabolites, including valine, leucine, isoleucine, and 
the sum of the above three amino acids [11-13]. Moreover, some prospective studies found that the plasma concentrations of BCAAs are prognostic for the onset and progress of type 2 diabetes in long-term follow-up [14, 15]. These indicated that BCAA, known as important nutrient signals, might play an important role in the pathogenesis of IR and T2DM [16]. In recent years, several studies have performed many investigations on BCAA and IR as well as diabetes risks across different races, sex, and so on. The results have shown big differences in the BCAA level due to variances in ethnicity, sex, gene expressions, and dietary pattern. However, studies focusing on phenotypic and genetic factors influencing BCAA level are largely lacking. Thus, this systematic review aims to evaluate the relationship between BCAA and insulin resistance as well as later diabetes risks and explore the phenotypic and genetic factors influencing BCAA level and its relationship with IR based on the available studies.

\section{Method}

2.1. Literature Search. We searched papers available on MEDLINE, EMASE, ClinicalTrials.gov, the Cochrane Library, and Web of Science for relevant studies from inception to March 2016. A search strategy was applied for MEDLINE according to medical subject headings ( $\left.\mathrm{MeSH}^{\circledR}\right)$ terms and some key words. Different possible variations and combinations of the following search terms were used: "metabolomic profiles", "metabolomic approach", "serum metabolites", "metabolic signature", "branched-chain amino acids", "BCAA", "leucine", "valine", "isoleucine", "amino acids metabolism", "insulin resistance", "nuclear magnetic spectroscopy," "NMR", and "mass spectrometry". Two persons (Xue Zhao and Qing Han) independently did the search process in order to minimize selection bias. If there were disagreements, a third person will join in and resolve disagreements by consensus (Guixia Wang).

2.2. Inclusion and Exclusion Criteria. Inclusion and exclusion criteria were set up before starting paper selection. The following inclusion criteria were applied: (1) all subjects should be adults, which means that age is $>18$ years and $<65$ years; (2) participants should be free of any thyroid or metabolic disorders requiring treatment such as diabetes, hypertension, severe dyslipidemia, and coronary heart disease; (3) studies need to include adiposity measures (such as BMI or waist circumference) and HOMA-IR according to the formula glucose $(\mathrm{mmol} / \mathrm{L}) \times$ insulin $(\mathrm{pg} / \mathrm{mL}) / 22.5$ [17], and serum BCAAs and related metabolites measurement results should be the outcome; (4) metabolomic techniques, such as MS, NMR spectroscopy, and UPLC, were applied to detect metabolite profiles in human blood; (5) principal components analysis (PCA) was used as a means to reduce the complexity of the variables; and (6) only papers published in English were included in our paper.

Studies were excluded if subjects were $<18$ or $>65$ years old, or were pregnant, or had diabetes, or had other chronic diseases, or had medication history. The metabolomics profiles extracted from urea are ruled out. Also, reviews, conference abstracts, case reports, and meta-analyses were excluded.

2.3. Data Extraction and Analysis. Data on population characteristics and metabolomic profiles were extracted. This procedure was done by two different persons (Xue Zhao and Qing Han). Because of differences in study design, method, and population characteristics, it is not appropriate to perform the quantitative meta-analysis.

BCAAs are known as essential amino acids, including valine, leucine, and isoleucine. Some included studies using BCAA to represent the sum of the above three amino acids. However, other studies point out the specific changes of valine, leucine, or isoleucine. Thus, in this review, we also use BCAA to represent the sum of three amino acids and each amino acid to represent itself in order to sustain the original state of included papers.

2.4. Methodological Quality Assessment. To assess the methodological quality of included studies, QUADOMICS tool was applied. QUADOMICS was developed to assess quality issues specific to "-omics" research, including the quality assessment of studies included in systematic reviews [18]. To differentiate high or low quality, studies will be scored from 1 to 16 . And studies which scored more than $11 / 16$ are regarded as "high quality," and studies which scored less than 11 are regarded as "low quality."

\section{Results}

3.1. Literature Search and Study Characteristics. After careful selection, 23 studies (including 20,091 participants) were included in our systematic review, which met the inclusion/exclusion criteria. Figure 1 presented the procedure of the literature selection based on PRISMA statement [19]. At first, 510 records were identified, and 94 full papers were retrieved. After reading all the papers, twenty-three unique studies providing adequate data were included in this systematic review.

Table 1 showed the details about general population characteristics of the twenty-three studies with 20,091 participants. The included studies were published between 2009 and 2016. Seven studies were conducted in the United States $[12,14,20-24]$, four studies were from Finland [25-28], three studies were from China [29-31], two studies were from Canada [32, 33] and Japan [34, 35], and one study was from each of the following countries: UK [36], Korea [37], Singapore [38], Germany [15], and Mexico [39]. The studies varied in sample size from 30 to 7098 with a median of 873. There were seventeen studies enrolling both males and females, and two studies focused on women only [26, 32], while the rest of the four studies address novel findings from males [31, 36-38]. The age of participants in our studies ranged from 18 to 59 years. All studies focused on the serum metabolites and insulin resistance (HOMA-IR). The analytical platforms used for metabolite detection included amino acid analyser, HPLC/fluorescence spectroscopy, ultraperformance LC-MS, GC-MS, FIA-MS/MS, UPLC-Q-TOF MS, 


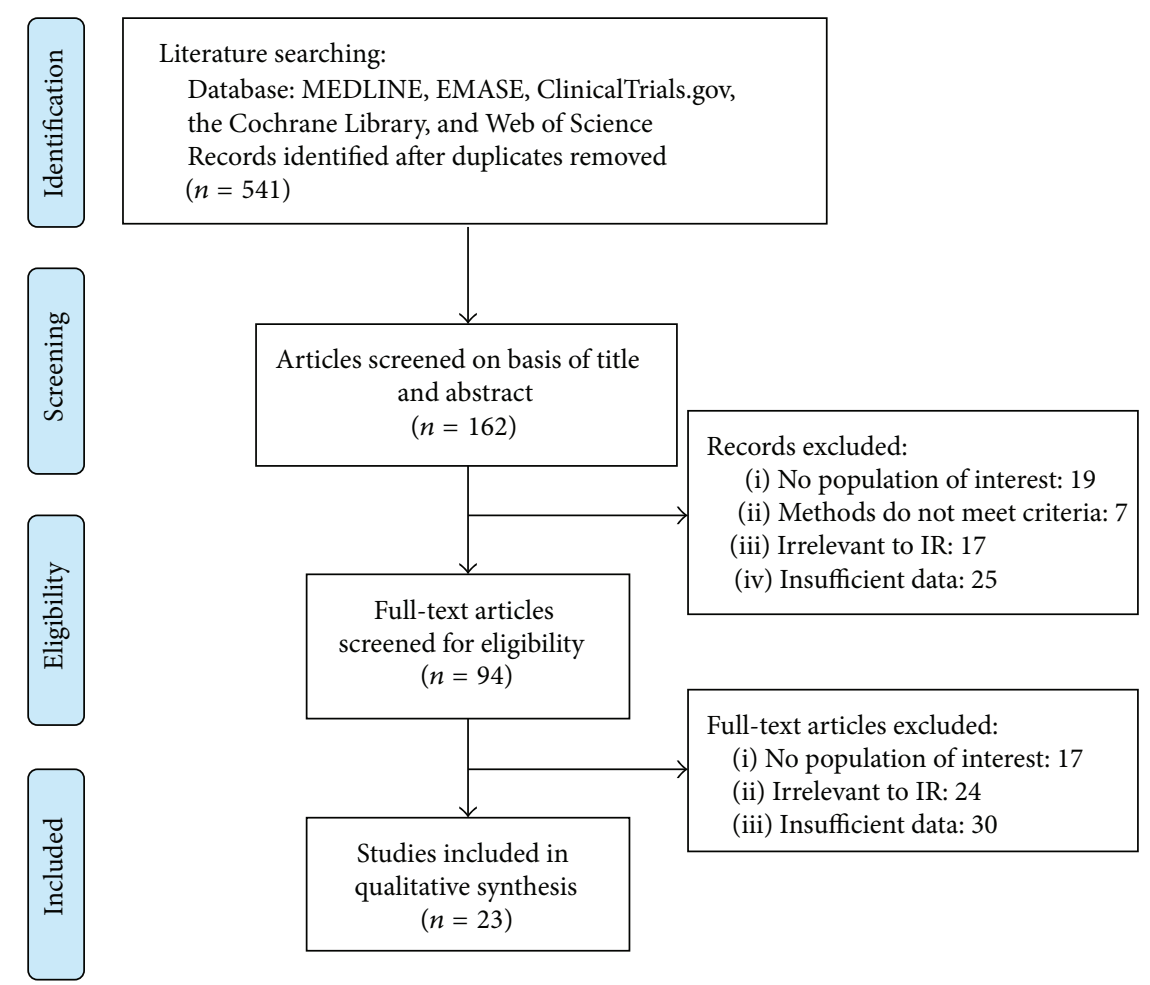

FIGURE 1: The flow chart of paper selection procedure in this systematic review.

NMR spectroscopy, CE-MS, ESI-LC-MS/MS, and UPLCTQ-MS.

3.2. BCAA and Insulin Resistance. Included studies revealed significant alterations of BCAA in obese adults and the intimate association between BCAA and IR. Newgard et al. [12] revealed that obese subjects presented 2.3-fold higher HOMA-IR than lean controls $(P<0.0001)$. Notably, some metabolites showed significant differences between obese and lean controls, which mainly refer to leucine/isoleucine, valine, the aromatic amino acids, and C3 and C5 acylcarnitines $(P<0.0001)$. Furthermore, a significant linear relationship was shown between these BCAA-related metabolites and HOMA-IR $(r=0.58, P<0.0001)$. Consistent results were shown in the study of Würtz et al. [28] on 7,098 young adults, comprising two Finnish cohorts, the Northern Finland Birth Cohort 1966 (NFBC) and the Cardiovascular Risk in Young Finns Study (YFS). They also showed that the BCAA together with other related metabolites was positively associated with HOMA-IR $(P<0.0005)$. Boulet et al. [32] suggested the positive relationship between AA related factors and HOMA-IR $(r=0.35, P<0.01)$. And compared with valine and isoleucine, leucine showed highest correlation with HOMA-IR $(r=0.26)$. Another recent twin study from Bogl et al. [25] consisting of 286 subjects (MZ: 136, DZ: 150) showed HOMA-IR correlated significantly with higher valine, leucine, and aromatic amino acids (AAA) as well as lipid profiles $(r=0.30-0.40)$. In the study by Tai et al. [38] on 263 nonobese Asian Indian and Chinese men, they divided enrolled subjects into low HOMA and high
HOMA groups; the results showed individuals with high HOMA presented higher level of valine $(P=0.0033)$ and leucine/isoleucine $(P=0.0321)$ in both Chinese and Asian Indians. Yamada et al. [35] showed the consistent results with previous studies in 94 nonobese Japanese people. Another study [34] on Japanese with normal glucose tolerance also showed positive correlation between HOMA-IR and valine, glutamate, and tyrosine levels, but negative correlation was found in citrulline, glutamine, and glycine levels.

Despite the altered BCAA level in peripheral circulation, BCAA metabolism in target tissue such as subcutaneous adipose tissue (SAT) and visceral adipose tissue (VAT) also showed significant changes in obese and insulin resistant patients [27, 32, 33]. Naukkarinen et al. [27] presented a close correlation between IR and downregulated mitochondrial BCAA catabolism in the adipose tissue of obese cotwins, who showed significantly lower insulin sensitivity and higher plasma insulin concentrations than their lean cotwins.

\subsection{Elevated BCAAs and Later Risk for Diabetes and Metabolic} Disorders. After evaluating all included studies, six prospective studies were found reporting the relationship between elevated BCAAs and later risk of type 2 diabetes [14, 15, $20,28,29,36]$. Wang et al's study on Framingham cohort [14] showed that 1 SD increment in five target amino acids (isoleucine, leucine, valine, tyrosine, and phenylalanine) was associated with a $57-102 \%$ increase in the risk of future diabetes $(P=0.0002-0.002)$. In addition, moderate association was found between baseline amino acids level and HOMAIR as well as HOMA-beta $(r=0.24-0.37, P<0.001)$. 


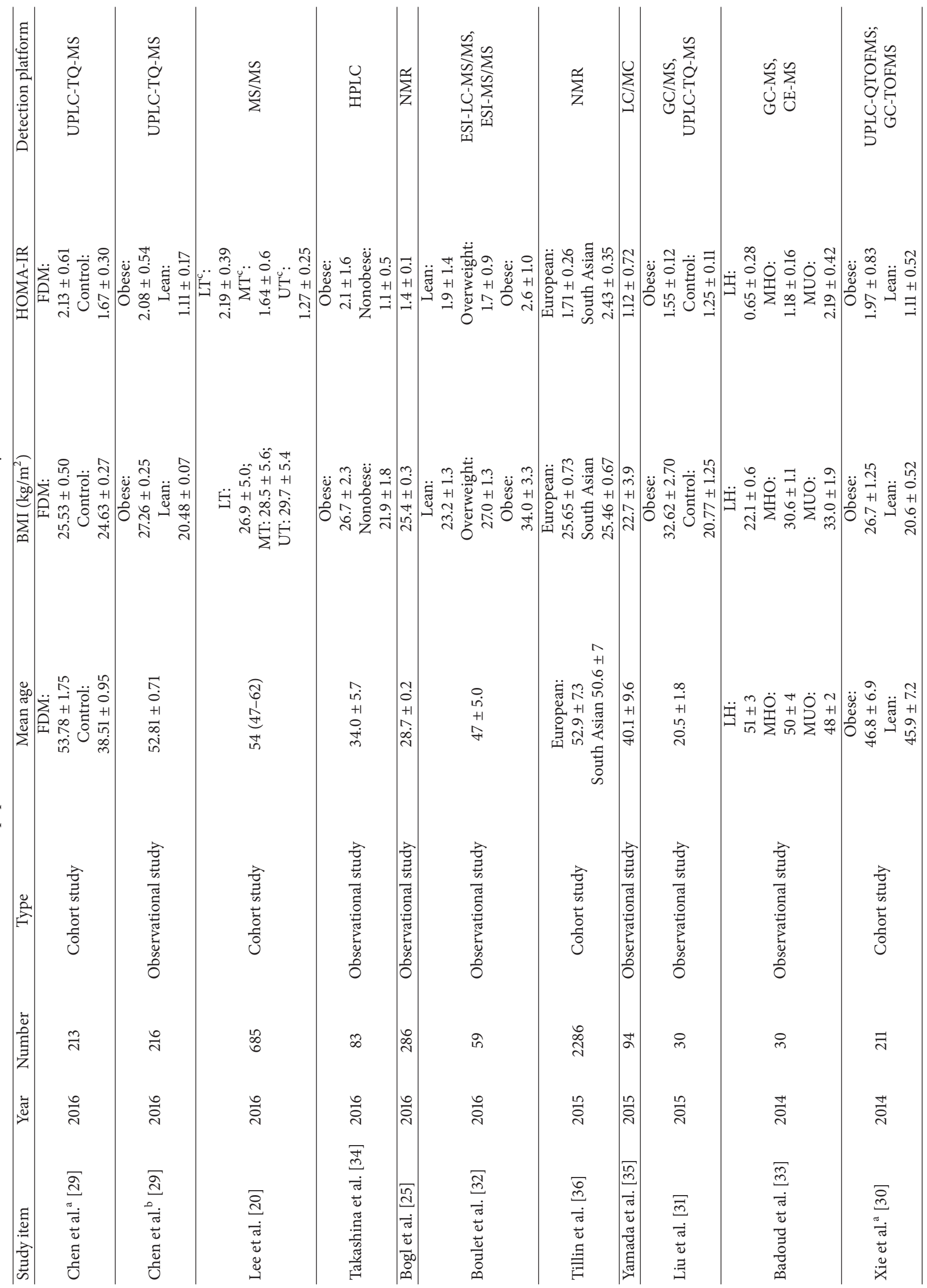




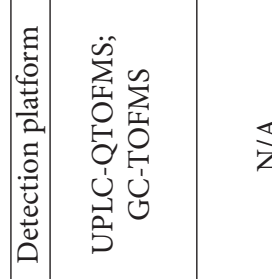

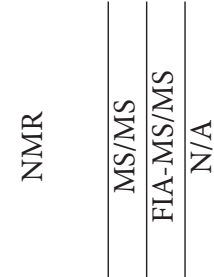

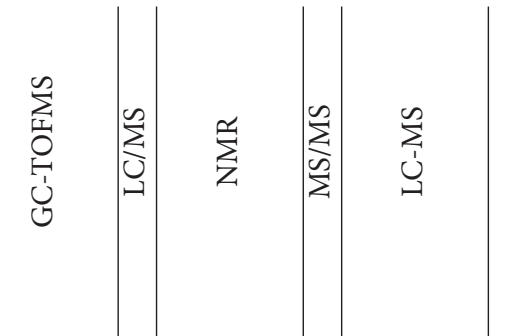

番

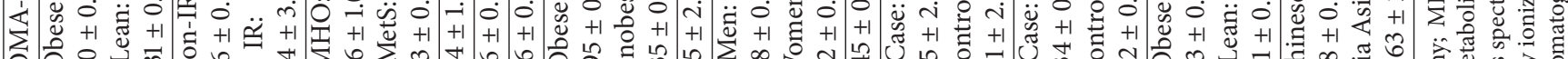

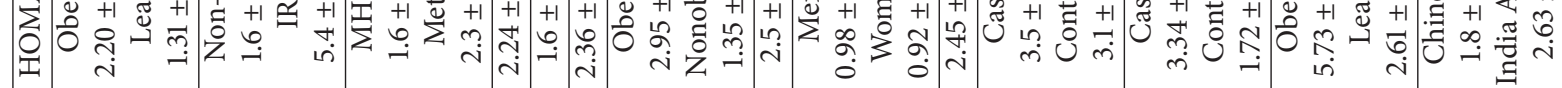

\section{范

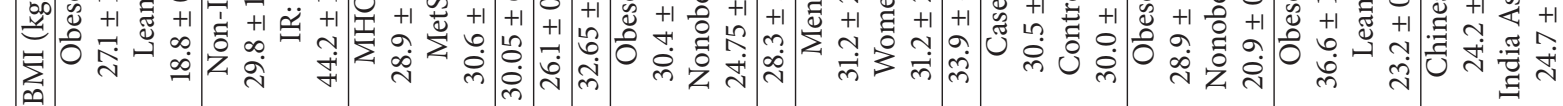

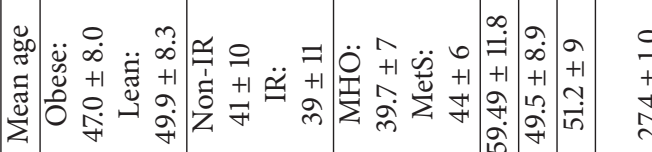

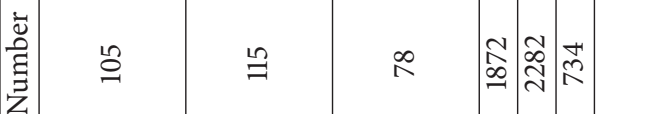

|

|

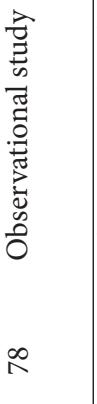

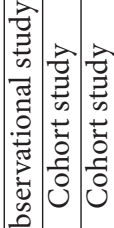

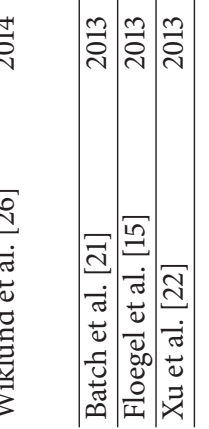

茫

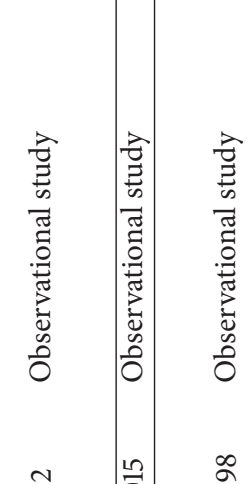

$\stackrel{0}{+}$
+1
$\stackrel{+}{4}$

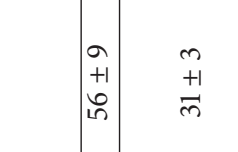

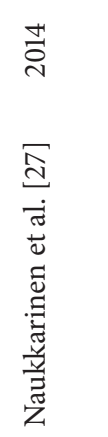

กี๊

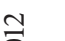

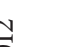

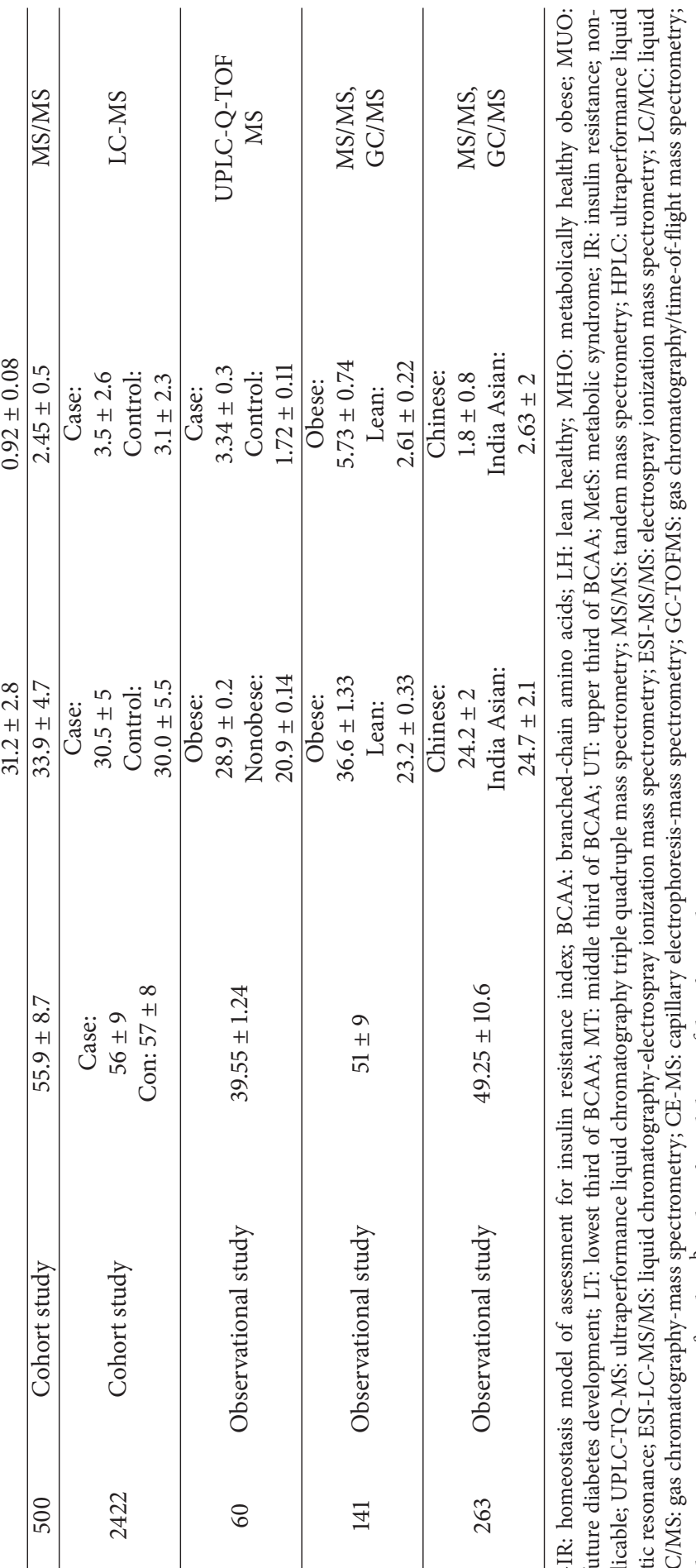

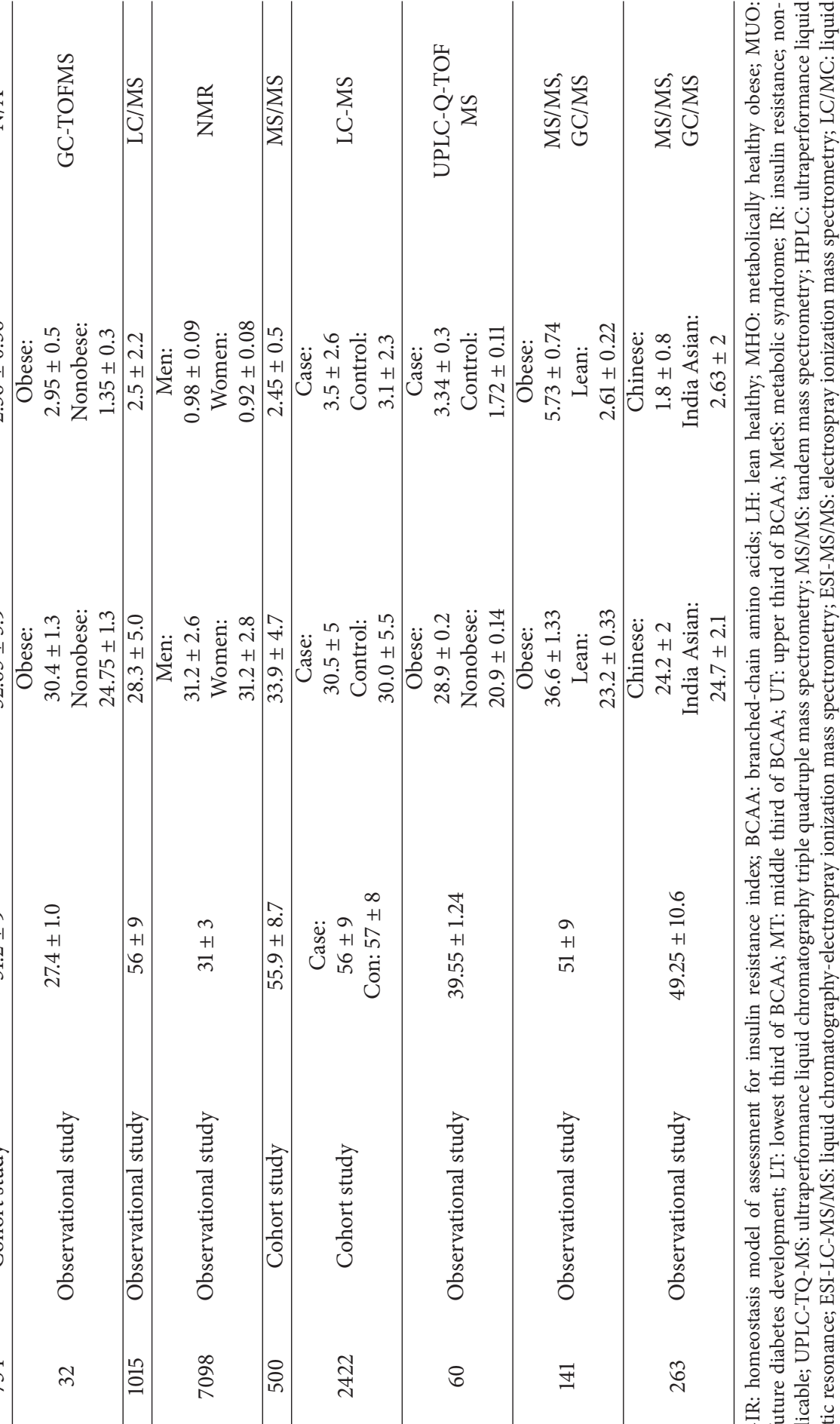

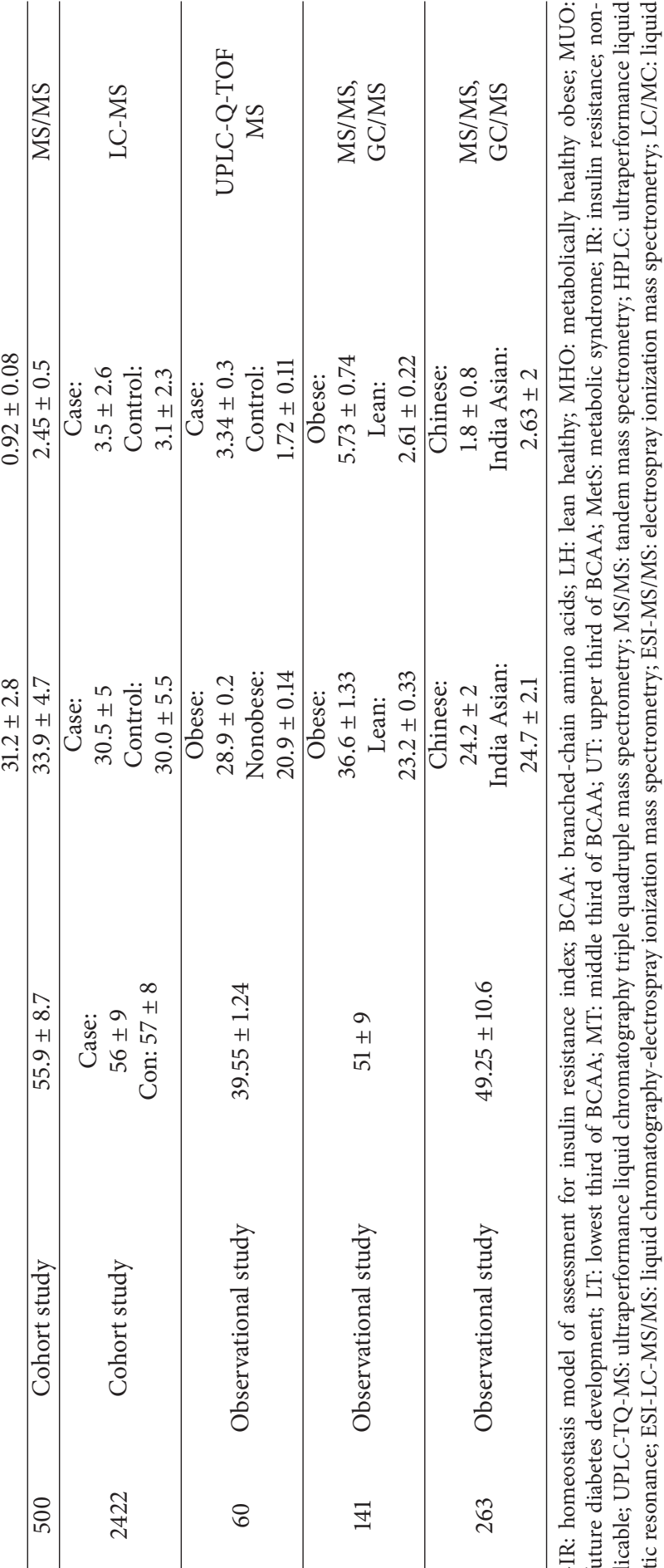

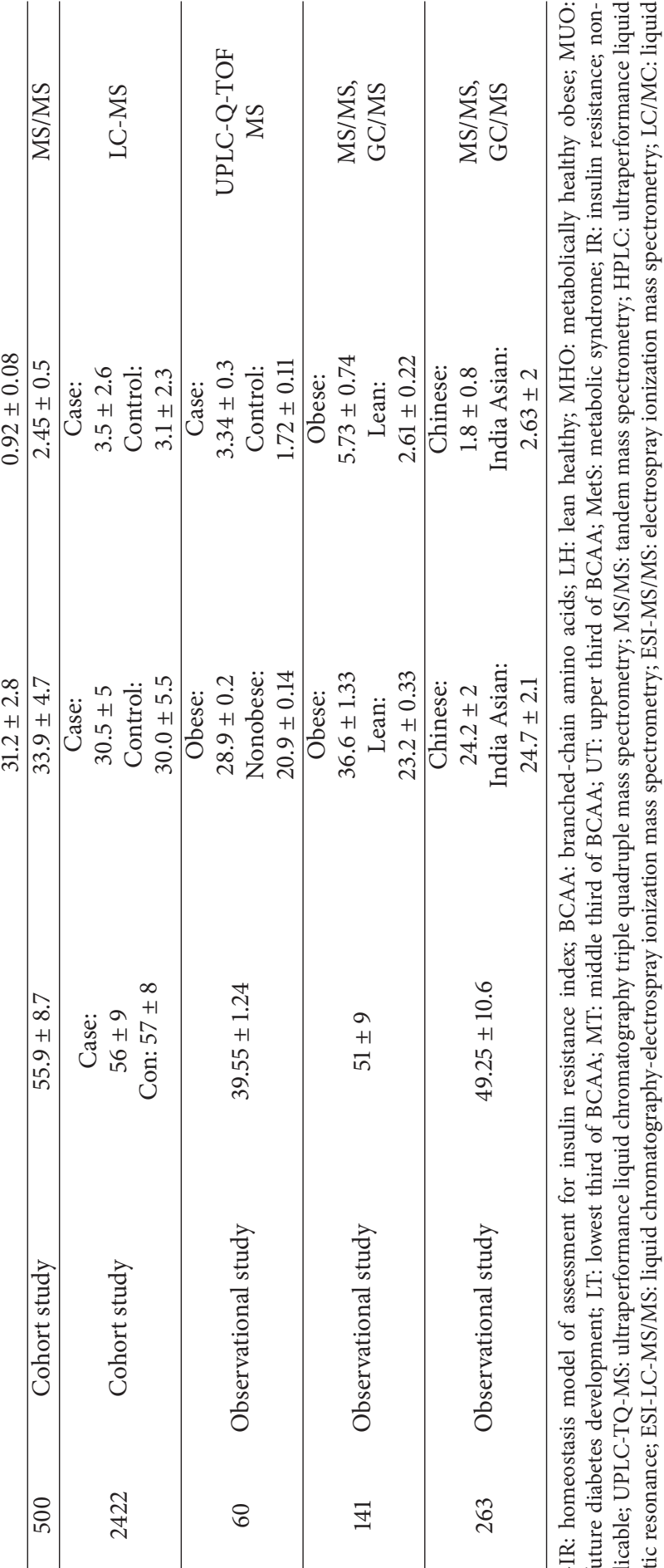

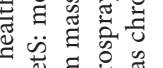

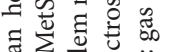
î

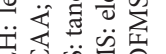

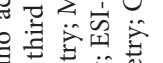
要要 पै क्षे

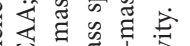

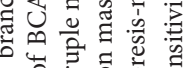

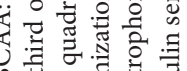
흘 常

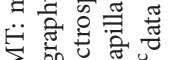

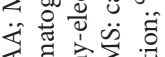

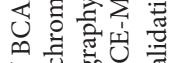
穴.

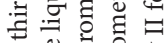
है च $\because$ 윤 $\because$ $\forall$ 嵌 客

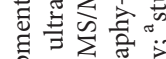
ô 它婳 过论部

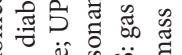

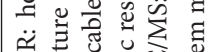

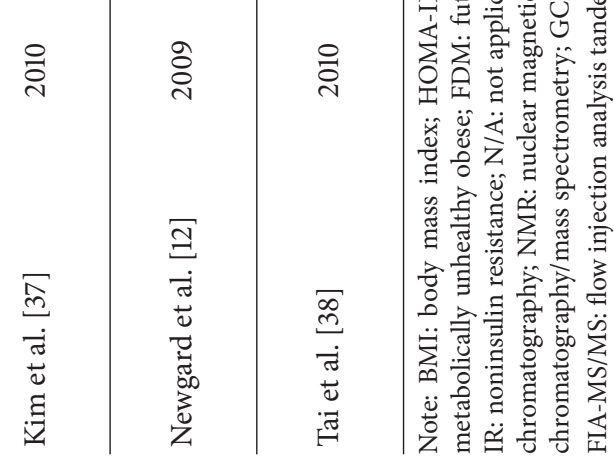


TABLE 2: The influence of race in BCAA level and its relationship with insulin resistance.

\begin{tabular}{lccll}
\hline Study & Country & Race & BCAA-related metabolites identified & $\begin{array}{l}\text { Relation between BCAA } \\
\text { and IR }\end{array}$ \\
\hline Lee et al. [20] & Canada & $\begin{array}{c}\text { Caucasians, } \\
\text { African Americans, } \\
\text { Hispanics }\end{array}$ & Valine, leucine, isoleucine, total BCAA & $\begin{array}{l}\text { Positive, } \\
\text { Caucasians plus Hispanics } \\
>\text { African Americans }\end{array}$ \\
\hline Tillin et al. [36] & Finland & $\begin{array}{c}\text { Europeans, } \\
\text { South Americans }\end{array}$ & $\begin{array}{l}\text { Isoleucine, leucine, valine, phenylalanine, } \\
\text { tyrosine, alanine, glutamine, glycine, histidine }\end{array}$ & $\begin{array}{l}\text { Positive, } \\
\text { South Americans }> \\
\text { Europeans }\end{array}$ \\
\hline Tai et al. [38] & Singapore & $\begin{array}{l}\text { Chinese, } \\
\text { Asian Indians }\end{array}$ & $\begin{array}{l}\text { Valine, leucine/isoleucine, phenylalanine, } \\
\text { tyrosine, glutamate/glutamine, ornithine, } \\
\text { alanine, proline }\end{array}$ & $\begin{array}{l}\text { Positive, } \\
\text { Chinese }>\text { Asian Indians }\end{array}$ \\
\hline
\end{tabular}

Note: IR: insulin resistance; BCAA: branched-chain amino acids.

Besides, similar results have been validated in another four large cohorts with long-term follow-up $[15,20,29,36]$. In Tillin et al.s study [36] on 801 European and 643 South Asian participants with 19-year follow-up, 227 South Asian men (35\%) and 113 European men (14\%) developed diabetes [36]. And amino acid metabolites (isoleucine, leucine, valine, phenylalanine, and tyrosine) were associated with incidence of diabetes in both ethnic groups after logistic regression analyses $\left(\mathrm{OR}_{\text {isoleucine }}=3.11 ; \mathrm{OR}_{\text {leucine }}=3.36 ; \mathrm{OR}_{\text {valine }}=\right.$ 3.34). In recent studies on Chinese population [29], 51 individuals presented similar level of metabolic markers but higher amino acids level (valine, leucine, isoleucine, tyrosine, and phenylalanine) and 162 controls were enrolled. After 10year follow-up, the positive correlation was found between the baseline five AAs and future diabetes incidents in these 51 individuals (ORs per SD $>1.5$ and $P<0.001$ ). However, the baseline metabolic markers failed to predict the risk of diabetes $(P>0.05)$. In the EPIC-Potsdam study with 7 year follow-up, Floegel et al. [15] showed positive correlation between isoleucine, valine, and future risk of T2DM (RR per SD 1.30 [95\% CI 1.17-1.43] and 1.27 [1.16-1.40], resp.). Also, the ethnical difference in using BCAA to predict diabetes is presented by Lee et al. [20]; the positive relationship with later diabetes risk was more obvious in Caucasians or in the combined Caucasian and Hispanic group but not in African Americans during 5-year follow-up. In a word, compared with already established metabolic factors, elevated BCAA level can largely improve the accuracy of prediction on future metabolic risk [15].

3.4. Factors Influencing BCAA Level and IR. Multiple factors have shown to influence the concentration of BCAA and its correlation with IR. Based on current evidence, these factors can be divided into two main aspects: phenotypic modification and genetic modification. Phenotypic part refers to race, gender, and dietary pattern; and genetic part refers to relative gene variants in BCAA metabolism.

3.4.1. Race Difference in BCAA and Its Relationship with IR. Three studies compared BCAAs outcomes across different races, including Europeans versus South Asians [36], Chinese versus Indian Asians [38], and Caucasians versus African
Americans versus Hispanics [20]. The detailed study characteristics about the influence of different race in BCAA level and its relationship with IR were shown in Table 2. Tillin et al. [36] reported that South Asian participants (1,279), compared with European $(1,007)$, had higher serum concentrations of isoleucine $(P<0.0001)$ but weaker correlation with obesity measurements. During 19-year follow-up in this study, higher risk of incident diabetes was also found in South Asian (34\%) compared to European subjects (14\%). Tai et al. [38] revealed that Chinese individuals with high HOMA had higher levels of amino acids (valine and leucine/isoleucine) than Chinese individuals with low HOMA $\left(P_{\text {valine }}=0.005\right.$ and $P_{\text {leucine } / \text { isoleucine }}=0.011$, resp.). Similar association was also observed in Asian Indians in other amino acids but did not reach statistical significance. The Insulin Resistance Atherosclerosis Study (IRAS) from Lee et al. [20] demonstrated the variances in ethnicity among Caucasians $(n=$ 290), Hispanics $(n=230)$, and African Americans $(n=$ 165). In stratified analysis by ethnicity, they found negative association between BCAA levels and insulin sensitivity $\left(S_{\mathrm{I}}\right)$ in Caucasians and Hispanics $[\beta$-coefficients: -0.0027 $(-0.0035,-0.0020)]$. After 5-year follow-up, positive correlation between BCAA and the risk of developing diabetes was only found in Caucasians and Hispanic group but not in African Americans. As for other races (such as Japanese, Chinese, and Finns), similar positive association between BCAA and IR was also presented $[29,30]$.

3.4.2. Gender Difference in BCAA and Its Relationship with IR. Seven studies addressed gender difference in BCAAs levels $[20,25,28,30,32,34,35]$. The detailed study characteristics about the comparison of altered BCAA level between male and female were shown in Table 3. The study on 7,098 young adults from Würtz et al. [28] displayed positive association between BCAA (leucine, isoleucine, and valine) and IR, and stronger correlation was found in men compared with women $\left(P_{\text {leucine }}<0.001 ; P_{\text {isoleucine }}=0.006 ; P_{\text {valine }}=0.001\right)$. In addition, a study from 94 Japanese people [35] revealed that BCAAs (leucine, isoleucine, valine) were positively associated with IR. However, this positive association was only found in women $(r=0.354, P=0.016)$, which did not reach statistical significance in men $(r=0.245, P=0.094)$, while, in a study on 685 participants, Lee et al. [20] found that men 
TABLE 3: Comparison of altered BCAA level between male and female.

\begin{tabular}{|c|c|c|c|c|c|}
\hline Study & Number & Men & Female & BCAA and related metabolites identified & $\begin{array}{l}\text { Relation between BCAA and } \\
\text { IR }\end{array}$ \\
\hline Lee et al. [20] & 685 & $45 \%$ & $55 \%$ & Valine, leucine, isoleucine, total BCAA & $\begin{array}{l}\text { Positive, } \\
\text { male }>\text { female }\end{array}$ \\
\hline Takashina et al. [34] & 83 & $80 \%$ & $20 \%$ & $\begin{array}{l}\text { Valine, isoleucine, leucine, lysine, methionine, } \\
\text { phenylalanine, threonine, tryptophan, alanine, } \\
\text { arginine, asparagine, } \alpha \text {-aminobutyric acid, citrulline, } \\
\text { cystine, glutamate, glutamine, glycine, ornithine, } \\
\text { proline, serine, taurine, tyrosine, histidine }\end{array}$ & $\begin{array}{l}\text { Positive, } \\
\text { male }>\text { female }\end{array}$ \\
\hline Boulet et al. [32] & 59 & $0 \%$ & $100 \%$ & $\begin{array}{l}\text { Leucine, histidine, isoleucine, lysine, methionine, } \\
\text { phenylalanine, threonine, tryptophan, valine, alanine, } \\
\text { arginine, citrulline, cystine, glycine, ornithine, proline, } \\
\text { serine, taurine, tyrosine, C3 }\end{array}$ & Positive (leucine) \\
\hline Yamada et al. [35] & 94 & $51 \%$ & $49 \%$ & $\begin{array}{l}\text { Valine, leucine, isoleucine, total BCAA, alanine, } \\
\text { tryptophan, phenylalanine, tyrosine, ornithine, glycine, } \\
\text { glutamate/glutamine, methionine, lysine, cysteine, } \\
\text { aspartic acid, total AA }\end{array}$ & $\begin{array}{l}\text { Positive, } \\
\text { male }>\text { female }\end{array}$ \\
\hline Xie et al. ${ }^{\mathrm{a}}[30]$ & 211 & $36 \%$ & $64 \%$ & $\begin{array}{l}\text { Valine, isoleucine, leucine, glutamic acid, tryptophan, } \\
\text { tyrosine, carnitine, phenylalanine, alanine, } \\
\text { beta-tyrosine, creatine }\end{array}$ & $\begin{array}{l}\text { Positive, } \\
\text { male }>\text { female }\end{array}$ \\
\hline Xie et al. ${ }^{b}[30]$ & 105 & $38 \%$ & $62 \%$ & $\begin{array}{l}\text { Valine, isoleucine, leucine, glutamic acid, tryptophan, } \\
\text { tyrosine, carnitine, phenylalanine, alanine, } \\
\text { beta-tyrosine, creatine }\end{array}$ & $\begin{array}{l}\text { Positive, } \\
\text { male }>\text { female }\end{array}$ \\
\hline Würtz et al. [28] & 7098 & $48 \%$ & $52 \%$ & $\begin{array}{l}\text { Leucine, isoleucine, valine, glutamine, pyruvate, } \\
\text { creatinine, alanine, phenylalanine, phospholipids, fatty } \\
\text { acids }\end{array}$ & $\begin{array}{l}\text { Positive, } \\
\text { male }>\text { female }\end{array}$ \\
\hline
\end{tabular}

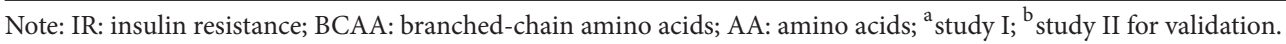

TABLE 4: The influence of dietary pattern and weight loss in BCAA level.

\begin{tabular}{lccll}
\hline Study & \multicolumn{1}{c}{ Diet } & Weight loss & BCAA and related metabolites identified & $\begin{array}{l}\text { Relationship with BCAA } \\
\text { and IR }\end{array}$ \\
\hline Batch et al. [21] & BCAA supplement & No & $\begin{array}{l}\text { Leucine/isoleucine, valine, tyrosine, methionine, } \\
\text { alanine, histidine, phenylalanine; C3; C5 }\end{array}$ & $\begin{array}{l}\text { Not related to BCAA and } \\
\text { IR }\end{array}$ \\
\hline Xu et al. [22] & High \& low fat & Yes & $\begin{array}{l}\text { Valine, isoleucine, leucine, tyrosine, phenylalanine; } \\
\text { tryptophan, BCAA/AAA }\end{array}$ & $\begin{array}{l}\text { Corresponded to gene } \\
\text { variants }\end{array}$ \\
\hline Würtz et al. [28] & Protein intake & No & $\begin{array}{l}\text { Leucine, isoleucine, valine, glutamine, pyruvate, } \\
\text { alanine, creatinine, phenylalanine, phospholipids, } \\
\text { fatty acids }\end{array}$ & $\begin{array}{l}\text { Positive for valine level } \\
\text { Not related to IR }\end{array}$ \\
\hline Shah et al. [24] & BCAA intake & Yes & $\begin{array}{l}\text { Alanine, leucine/isoleucine, valine, methionine, } \\
\text { phenylalanine, tyrosine, glutamate/glutamine, } \\
\text { ornithine }\end{array}$ & $\begin{array}{l}\text { Slightly positive for BCAA } \\
\text { level } \\
\text { Not related to IR }\end{array}$ \\
\hline Newgard et al. [12] BCAA supplement & No & $\begin{array}{l}\text { Alanine, valine, leucine/isoleucine, phenylalanine, } \\
\text { tyrosine, glutamate/glutamine, aspartate/asparagine }\end{array}$ & $\begin{array}{l}\text { Positive for BCAA and IR } \\
\text { (in rats) }\end{array}$ \\
\hline
\end{tabular}

Note: IR: insulin resistance; BCAA: branched-chain amino acids; AAA: aromatic amino acids.

had higher level of BCAA as well as BMI, waist-hip ratio, IR, and dietary energy intake compared with women. The gender-dependence was also reported by Xie et al. [30]. They presented that BCAA was correlated with IR only in obese Chinese men; similar results were validated by another two cohorts on Chinese and Americans. Consistent results were also presented by Takashina et al. [34]. Other studies did not show difference in BCAA or IR between male and female.

3.4.3. Dietary Pattern and Weight Loss in BCAA and Their Relationship with IR. Five studies showed the relationship between diet and BCAA $[12,21,22,24,28]$. The detailed study characteristics about the influence of dietary pattern and weight loss in BCAA level were shown in Table 4 . In the study of Shah et al. [24], BCAAs (valine and leucine/isoleucine) not only correlated positively with baseline HOMA-IR $(r=$ $0.50, P<0.0001)$ but also correlated with 6 -month HOMAIR $(r=0.28, P<0.0001)$ as well as $\Delta$ HOMA-IR $(r=$ $-0.38, P<0.0001)$ after weight loss. Moreover, during the weight-loss period, valine and leucine/isoleucine presented remarkable reduction $(P=0.005, P<0.0001)$. To test the influence of BCAA intake on plasma BCAA level, they 
TABLE 5: BCAA-related gene variants in specific tissues based on included studies.

\begin{tabular}{|c|c|c|c|}
\hline Study & BCAA and related metabolites identified & Identified gene variants & Specific tissue \\
\hline Boulet et al. [32] & $\begin{array}{l}\text { Leucine, histidine, isoleucine, lysine, methionine, } \\
\text { phenylalanine, threonine, tryptophan, valine, } \\
\text { alanine, arginine, citrulline, cystine, glycine, } \\
\text { ornithine, proline, serine, taurine, tyrosine, C3 }\end{array}$ & $\begin{array}{l}\text { BCKDHA, BCKDHB, } \\
\text { BCAT1, BCAT2, BCKDK }\end{array}$ & $\begin{array}{l}\text { Visceral adipose tissue, } \\
\text { subcutaneous adipose } \\
\text { tissue }\end{array}$ \\
\hline Badoud et al. [33] & $\begin{array}{l}\text { Valine, isoleucine, ornithine, alanine, aspartic } \\
\text { acid, glutamine, phenylalanine, methionine, } \\
\text { tyrosine, glycine, cysteine, aspartic acid, } \\
\text { glutamine/glutamic acid }\end{array}$ & $\begin{array}{l}B C A T 2, B C K D H A \\
B C K D H B, D B T\end{array}$ & $\begin{array}{l}\text { Subcutaneous adipose } \\
\text { tissue }\end{array}$ \\
\hline Serralde-Zúñiga et al. [39] & Total BCAA, isoleucine, leucine, valine & $\begin{array}{l}\text { BCAT2, BCKDH E1a, } \\
\text { SREBP-1, FTO }\end{array}$ & Omental adipose tissue \\
\hline Xu et al. [22] & $\begin{array}{l}\text { Valine, isoleucine, leucine, tyrosine, } \\
\text { phenylalanine, tryptophan, BCAA/AAA }\end{array}$ & PPM1K (rs 1440581), C allele & Blood \\
\hline Naukkarinen et al. [27] & $\begin{array}{l}\text { Leucine, isoleucine, valine, alanine, } \\
\text { phenylalanine, tyrosine, glutamine, } \\
\text { glutamate/glutamine }\end{array}$ & $\begin{array}{l}\text { Genes of BCAA catabolism } \\
\text { (monozygotic twin study) }\end{array}$ & $\begin{array}{l}\text { Subcutaneous adipose } \\
\text { tissue }\end{array}$ \\
\hline Würtz et al. [28] & $\begin{array}{l}\text { Leucine, isoleucine, valine, glutamine, pyruvate, } \\
\text { creatinine, alanine, phenylalanine, phospholipids, } \\
\text { fatty acids }\end{array}$ & GCKR (rs 1260326) & Blood \\
\hline
\end{tabular}

Note: IR: insulin resistance; BCAA: branched-chain amino acids; AAA: aromatic amino acids; BCKDK: branched-chain keto acid dehydrogenase kinase; $B C K D H E 1 a$ (BCKDHA): branched-chain keto acid dehydrogenase E1, alpha polypeptide; $B C K D H B$ : branched-chain keto acid dehydrogenase E1 subunit beta; $B C A T 1$ : branched-chain amino acid transaminase 1; $B C A T 2$ : branched-chain amino acid transaminase 2; $D B T$ : dihydrolipoamide branched-chain transacylase E2; SREBP-1: sterol regulatory element binding protein 1; FTO: fat mass and obesity associated; PPM1K: protein phosphatase, $\mathrm{Mg} 2+/ \mathrm{Mn} 2+$ dependent $1 \mathrm{~K}$; GCKR: glucokinase (hexokinase 4) regulator.

performed baseline and 6-month intake of BCAA in enrolled participants. In Shah et al.s study, BCAA intake refers to a sum of dietary intake on valine, leucine, and isoleucine intake. The results showed that BCAA intake was weakly related to peripheral BCAA level $(r=0.14, P=0.003)$, but change in BCAA intake was not correlated with plasma BCAA level $(P=0.39)$ and $\triangle$ HOMA-IR $(P=0.82)$. The study from Würtz et al. [28] demonstrated that dietary intake, especially for protein intake, was related to fasting metabolites levels (valine, phenylalanine, and tyrosine). However, no relationship was found in protein intake and IR. Batch et al. also presented no difference in glucose metabolism and HOMA-IR between controls and BCAA-supplement group [21]. However, study from Newgard et al. [12] on rats showed positive relationship in BCAA intake and IR, indicating the existence of species difference.

3.4.4. Gene Variants in BCAA and Its Relationship with IR. There were six studies reporting relevant gene variants in BCAA metabolism [22, 27, 28, 32, 33, 39]. The detailed study characteristics about BCAA-related gene variants in specific tissues were shown in Table 5. In study of Badoud et al. [33], plasma valine and isoleucine were positively correlated with HOMA-IR [lean healthy $(\mathrm{LH})<$ metabolic healthy obese $(\mathrm{MHO})<$ metabolic unhealthy obese (MUO)]. To explore the alterations in BCAA-related gene expression, they found downregulations of BCAA catabolism and TCA cycle in subcutaneous adipose tissue (SAT) from $\mathrm{MHO}$ and MUO patients compared with LH patients. The key mitochondrial genes involved in BCAA catabolism were significantly influenced, including the increase in BCAT1 and decrease in BCKDHA as well as BCAT2 in obese group compared with lean group. In two obese groups, MUO group had more significant reduction in BCAT2 and BCKDHA than $\mathrm{MHO}$ group. In other studies, Serralde-Zúñiga et al. [39] revealed significant reduction in the gene expressions of BCAT2 and BCKDH E1a in omental adipose tissue of insulin resistant subjects. Similar changes of gene expression were reported by Boulet et al. [32] in visceral adipose tissue (VAT) in obese women with BMI $>30 \mathrm{~kg} / \mathrm{m}^{2}(P<0.05)$, while, in SAT, only expression of BCAT2 was decreased. Naukkarinen et al. [27] studied gene variants in weightdiscordant monozygotic twins and found that genes related to BCAA catabolism, oxidative phosphorylation, and fatty acid $\beta$ oxidation were downregulated in SAT of obese cotwins compared with their lean counterpart. Due to the impairment in BCAA clearance, higher BCAA level was found in obese cotwins $(P=0.016)$. Another study from Würtz et al. [28] presented the positive correlation between metabolites (isoleucine, alanine, total fatty acids, etc.) and SNP rs1260326 in $G C K R$ which was associated with $\operatorname{IR}(P=0.001)$. Xu et al. [22] found that individuals carrying $C$ allele of the branchedchain amino acid/aromatic amino acid (BCAA/AAA) ratioassociated variant rs1440581 near PPM1K gene may benefit less during weight loss than those without this allele when undertaking an energy-restricted high-fat diet.

3.5. Quality Assessment. The study team conducted quality assessment process through QUADOMICS tool [18, 40]. In all the included studies, sixteen studies were defined as high quality, fulfilling more than 11 scores. Two different persons double-checked the general characteristics of included studies (Xue Zhao and Qing Han). 


\section{Discussion}

4.1. BCAA Metabolism and Its Correlation with IR. Leucine, isoleucine, and valine are known as BCAA. The metabolism of BCAA has been known as a significant factor in the pathophysiology of many multifactorial diseases, such as metabolic syndrome, cancer, and hepatic disease [41]. The catabolism of BCAA contains two important steps. The first step is a reversible transamination reaction converting BCAA to branched-chain a-keto acids (BCKAs). In this procedure, the enzyme branched-chain amino acid aminotransferase (BCAT) played an important role. At the same time, the other partner pair a-ketoglutarate/glutamate takes place with this transamination reaction. The next step in the BCAAs catabolism is irreversible, mediated by the mitochondrial branched-chain keto-acid dehydrogenase complex (BCKDC). This enzymatic step confers oxidation on the BCKAs, leading to the production of $\mathrm{NADH}, \mathrm{CO}_{2}$, and different end-products [42].

Changes in $B C A T$ and $B C K D C$ can have significant effects on BCAA catabolism $[43,44]$. Because of BCAA dysmetabolism, some potentially toxic intermediates in BCAA catabolic pathway might be accumulated, leading to the impaired cellular or organ function. In some studies, humans or animal models with impaired BCAA metabolism presented higher susceptibility to IR and T2DM compared with controls [41]. Olson et al. [43] showed that high level of toxic BCAA metabolites, rather than BCAAs themselves, could result in mitochondrial dysfunction and apoptosis in $\beta$-cell.

However, the mechanism between BCAA and IR remains unclear [45]. Studies have shown that persistent high nutrient signaling might lead to the onset of IR mediated by mTORC1 signaling pathway $[16,46]$. In recent years, mTOR has been considered as the important link between amino acids and insulin action. Once mTOR has been stimulated, it will activate $\mathrm{p} 70$ ribosomal S6 kinase ( $\mathrm{p} 70 \mathrm{~S} 6 \mathrm{~K})$. p70S6K regulates protein synthesis cascade and subsequently leads to the phosphorylation of its downstream target, ribosomal protein S6 kinase (S6K) [44, 47]. Moreover, activation of S6K1 leads to serine/threonine phosphorylation and therefore the inhibition of insulin receptor substrate (IRS)-1. This procedure not only impacts normal insulin signaling but also causes the degradation of IRS-1 [48]. However, much more studies are required in the future to figure out the role of $\mathrm{mTOR}$ pathway in elevated BCAA and IR.

4.2. Factors Influencing BCAA and Its Relationship with Insulin Resistance. The factors influencing BCAA level and its relationship with IR in this study mainly refer to race, gender, diet, and gene variants. First of all, after generating available studies on different ethnicities, we found that Asian people (Chinese or Japanese) seemed to be more susceptible to higher HOMA-IR and higher BCAA level compared with western people such as Caucasians or Europeans. Although Asian people usually hold lower BMI, studies have shown their higher susceptibility to impaired islet function at the early stages of metabolic disorders [29], while, in studies focusing on western people, Hispanics and Caucasians seemed to have stronger positive association between BCAA and IR than African Americans. Thus, the elevated BCAA level and its relationship with IR seem to be race-dependent. Future studies should extend their sample size in different races in order to validate the above findings. For gender difference, majority of included studies showed obese men had higher BCAA and stronger positive relationship with IR compared with female. This indicated future studies should pay much attention to the gender difference during data analysis and study design. In a word, diverse race difference and gender difference can have significant effects on BCAAs level and insulin resistant state, which should also be validated in studies with larger population.

The influence of dietary pattern on BCAA level and IR was not established yet. For a long time, beneficial effects were presented on the regulation of body weight, muscle protein synthesis, and glucose homeostasis in patients or animals applying BCAA supplementation or BCAA-rich diets $[49,50]$. In this study, the results showed that a BCAArich diet might have a weakly positive impact in peripheral BCAA level, which was most frequently explained by the insulinotropic properties of amino acids [51] or the decreased glycemic load of high-protein diets. Also, Xu et al's study [22] presented a new gene-diet interaction, illustrating that patients with $\mathrm{C}$ allele of BCAA/AAA might lose less weight during a weight-loss diet. As for the relationship between BCAA supplement and IR, most studies showed negative results, indicating that the ectogenic BCAA intake constituted an extremely small portion in the onset of IR. However, how BCAAs influence insulin signaling remains unclear. Thus, the relationship between diet, circulating BCAA, and IR deserves further exploration.

Although studying blood metabolites can uncover novel markers of potential clinical relevance, it is difficult to determine the relevant gene variants and specific tissues underlying changes in metabolic pathways. As mentioned, $B C A T$ and the BCKD complex in BCAA catabolism are broadly expressed in human tissues, indicating that extensive tissues hold the ability of BCAA's transamination and oxidation [13]. Among these tissues, skeletal muscle, adipose tissue, and liver seem to play the central role [45]. In our study, we mainly talked about the gene expressions of BCAA metabolism in adipose tissue based on included studies and revealed the significant role of gene expression changes in BCAA catabolism of adipose tissue regarding pathogenesis of IR. Consistent results were also reported by Pietiläinen et al. [52] and Solini et al. [53]. Another study validating the function of adipose tissue in peripheral BCAA level was performed by Herman et al. [54]. They showed that the transplanted adipose tissue had the capability of catabolizing circulating BCAAs in vivo and indicated the possibility that adipose tissue modulates circulating BCAA levels through regulating BCAA enzymes. All these showed that genetic variants of BCAA catabolism in adipose tissue might have important influences on altered BCAA level and the presence of IR to some extent.

In addition to adipose tissue, several studies have demonstrated the existence of BCAA dysmetabolism in other tissues, while few studies detected relative gene expressions. Shin et al. [55] showed that decreased hepatic $B C K D H$ 
might contribute to the increased plasma BCAAs. Moreover, hypothalamus presented an important role in impaired BCAA metabolism in obesity and diabetes. Their study on rats revealed that short-term overfeeding impaired brain insulin ability to lower BCAAs, which was also associated with reduced $B C K D H$ protein in liver. In addition, in a study of 35 subjects, Chevalier et al. [56] revealed the increased postabsorptive protein catabolism with elevated BCAA in obesity was associated with increased gluconeogenesis and led to dysglycemia. In skeletal muscle, Krebs et al. [57] reported that elevated plasma amino acid can induce IR in skeletal muscle through inhibiting the glucose transport/phosphorylation and later glycogen synthesis. As for gut microbiota, the amino acid fermenting bacteria in human gastrointestinal tract present the important role in the digestion and absorption of protein and amino acids [58, 59]. Beyond that, gut bacteria themselves also hold a higher level of BCAA-related amino acids [60]. However, it remains to be known if BCAA from gut bacteria can impact the homeostasis of BCAA in the host and how they influence each other. Future studies should focus on the BCAA catabolic alteration in different tissues and explore the mechanism of altered BCAA and its relationship with IR.

4.3. Prospects in BCAA and IR Treatment. BCAAs (valine, leucine, and isoleucine) can be an important and useful biomarker of IR. Besides the reflection of insulin resistant state, BCAA can also give feedback of drug effect. In a randomized, double-blind, controlled study on 25 overweight/obese adults [61], patients using insulin sensitizer therapy showed improved insulin sensitivity and reduced functional metabolites (BCAA) compared to placebo treatment after three months of intervention. Walford et al. [62] found administration of glipizide and metformin can influence the ratio-BCAA/AAAs acutely, and the magnitude of change was dependent on the IR status of participants. The above results demonstrate that BCAA or BCAA/AAAs may be useful biomarkers for monitoring the early response to therapeutic interventions in T2DM patients. Since protein intake or BCAA supplement will not influence plasma BCAA level too much, we highly recommend measurement of BCAA levels in order to capture the whole course of disease progress, including obesity, insulin resistant state, T2DM, and later drug effect. Improvement in this field will definitely deepen our understanding that BCAA (as a biomarker) can reflect not only IR but also pharmacological effects of drug intervention.

4.4. Strength and Limitation. Based on current studies, we found that BCAAs could be useful biomarker for IR and predictor for later diabetes risks. To our knowledge, this is the first review focusing on the relationship between BCAA and IR as well as the factors influencing BCAA level. All the eligible studies were published between 2009 and 2016, manifesting the latest development in this field. Although much more studies are largely needed, our study sheds light on the differences in ethnicity, sex, gene expression, and dietary pattern, which are important factors influencing BCAA level that should be controlled and considered when conducting clinical or basic studies. However, several limitations should be addressed here. There were huge heterogeneities in subject and study design; the control group and experimental group were not consistent across studies. This limitation restricts further comparison and data analyzed for meta-analysis.

\section{Conclusion}

In conclusion, this systematic review highlighted BCAAs (valine, leucine, and isoleucine) as useful biomarkers for early detection and diagnosis of IR in nondiabetic patients with obesity and as a valuable predictor for later risk of T2DM. Based on included studies, we found that differences in ethnicity, sex, gene expression, and dietary pattern can influence BCAA level and its relationship with IR. Future studies should focus on the mechanism of BCAA and IR in different tissues and explore the potential treatment targets for obesity and T2DM. Despite the long-time exploration of BCAA and its applications so far, much more studies are still required to bring it to light.

\section{Competing Interests}

No competing interests were declared.

\section{Authors' Contributions}

Xue Zhao and Guixia Wang designed this review; Xue Zhao and Qing Han conducted the paper selection; Chenglin Sun, Xiaokun Gang, and Yujia Liu performed the statistical analysis and data extraction; Xue Zhao and Guixia Wang wrote the manuscript and were responsible for the final content of the manuscript. All authors read and approved the final manuscript.

\section{Acknowledgments}

This work was supported by grants (belonging to Guixia Wang) from the Ministry of Education, Science and Technology Development Center (3M213BR43428) and the Science Technology Department of Jilin Province (3D511Z933428). It was also supported by grants (belonging to Xue Zhao) from Graduate Innovation Fund of Jilin University (2016042).

\section{References}

[1] P. Campos, A. Saguy, P. Ernsberger, E. Oliver, and G. Gaesser, "The epidemiology of overweight and obesity: public health crisis or moral panic?" International Journal of Epidemiology, vol. 35, no. 1, pp. 55-60, 2006.

[2] M. Arnold, N. Pandeya, G. Byrnes et al., "Global burden of cancer attributable to high body-mass index in 2012: a population-based study," The Lancet Oncology, vol. 16, no. 1, pp. 36-46, 2015.

[3] G. F. Lewis, A. Carpentier, K. Adeli, and A. Giacca, "Disordered fat storage and mobilization in the pathogenesis of insulin resistance and type 2 diabetes," Endocrine Reviews, vol. 23, no. 2, pp. 201-229, 2002. 
[4] C. Morris, C. O’Grada, M. Ryan et al., "The relationship between BMI and metabolomic profiles: a focus on amino acids," Proceedings of the Nutrition Society, vol. 71, no. 4, pp. 634$638,2012$.

[5] American Diabetes Association, "Diagnosis and classification of diabetes mellitus," Diabetes Care, vol. 32, pp. S62-S67, 2009.

[6] M. J. Gibney, M. Walsh, L. Brennan, H. M. Roche, B. German, and B. van Ommen, "Metabolomics in human nutrition: opportunities and challenges," The American Journal of Clinical Nutrition, vol. 82, no. 3, pp. 497-503, 2005.

[7] O. Y. Kim, J. H. Lee, and G. Sweeney, "Metabolomic profiling as a useful tool for diagnosis and treatment of chronic disease: focus on obesity, diabetes and cardiovascular diseases," Expert Review of Cardiovascular Therapy, vol. 11, no. 1, pp. 61-68, 2013.

[8] R. Pallares-Méndez, C. A. Aguilar-Salinas, I. Cruz-Bautista, and L. Del Bosque-Plata, "Metabolomics in diabetes, a review," Annals of Medicine, vol. 48, no. 1-2, pp. 89-102, 2016.

[9] S. E. Park, C.-Y. Park, and G. Sweeney, "Biomarkers of insulin sensitivity and insulin resistance: past, present and future," Critical Reviews in Clinical Laboratory Sciences, vol. 52, no. 4, pp. 180-190, 2015.

[10] D. K. Layman, "The role of leucine in weight loss diets and glucose homeostasis," The Journal of Nutrition, vol. 133, no. 1, pp. 261S-267S, 2003.

[11] M. S. Klein and J. Shearer, "Metabolomics and type 2 diabetes: translating basic research into clinical application," Journal of Diabetes Research, vol. 2016, Article ID 3898502, 10 pages, 2016.

[12] C. B. Newgard, J. An, J. R. Bain et al., "A branched-chain amino acid-related metabolic signature that differentiates obese and lean humans and contributes to insulin resistance," Cell Metabolism, vol. 9, no. 4, pp. 311-326, 2009.

[13] M. M. Adeva, J. Calviño, G. Souto, and C. Donapetry, "Insulin resistance and the metabolism of branched-chain amino acids in humans," Amino Acids, vol. 43, no. 1, pp. 171-181, 2012.

[14] T. J. Wang, M. G. Larson, R. S. Vasan et al., "Metabolite profiles and the risk of developing diabetes," Nature Medicine, vol. 17, no. 4, pp. 448-453, 2011.

[15] A. Floegel, N. Stefan, Z. Yu et al., "Identification of serum metabolites associated with risk of type 2 diabetes using a targeted metabolomic approach," Diabetes, vol. 62, no. 2, pp. 639-648, 2013.

[16] B. C. Melnik, "Leucine signaling in the pathogenesis of type 2 diabetes and obesity," World Journal of Diabetes, vol. 3, no. 3, pp. 38-53, 2012.

[17] D. R. Matthews, J. P. Hosker, A. S. Rudenski, B. A. Naylor, D. F. Treacher, and R. C. Turner, "Homeostasis model assessment: insulin resistance and $\beta$-cell function from fasting plasma glucose and insulin concentrations in man," Diabetologia, vol. 28, no. 7, pp. 412-419, 1985.

[18] B. Lumbreras, M. Porta, S. Márquez, M. Pollán, L. A. Parker, and I. Hernández-Aguado, "QUADOMICS: an adaptation of the Quality Assessment of Diagnostic Accuracy Assessment (QUADAS) for the evaluation of the methodological quality of studies on the diagnostic accuracy of '-omics'-based technologies," Clinical Biochemistry, vol. 41, no. 16-17, pp. 1316-1325, 2008.

[19] A. Liberati, D. G. Altman, J. Tetzlaff et al., "The PRISMA statement for reporting systematic reviews and meta-analyses of studies that evaluate healthcare interventions: explanation and elaboration," British Medical Journal, vol. 339, Article ID b2700, 2009.
[20] C. C. Lee, S. M. Watkins, C. Lorenzo et al., "Branched-chain amino acids and insulin metabolism: The Insulin Resistance Atherosclerosis Study (IRAS)," Diabetes Care, vol. 39, no. 4, pp. 582-588, 2016.

[21] B. C. Batch, S. H. Shah, C. B. Newgard et al., "Branched chain amino acids are novel biomarkers for discrimination of metabolic wellness," Metabolism: Clinical and Experimental, vol. 62, no. 7, pp. 961-969, 2013.

[22] M. Xu, Q. Qi, J. Liang et al., "Genetic determinant for amino acid metabolites and changes in body weight and insulin resistance in response to weight-loss diets: the preventing overweight using novel dietary strategies (POUNDS LOST) trial," Circulation, vol. 127, no. 12, pp. 1283-1289, 2013.

[23] S. Cheng, E. P. Rhee, M. G. Larson et al., "Metabolite profiling identifies pathways associated with metabolic risk in humans," Circulation, vol. 125, no. 18, pp. 2222-2231, 2012.

[24] S. H. Shah, D. R. Crosslin, C. S. Haynes et al., "Branched-chain amino acid levels are associated with improvement in insulin resistance with weight loss," Diabetologia, vol. 55, no. 2, pp. 321330, 2012.

[25] L. H. Bogl, S. M. Kaye, J. T. Rämö et al., "Abdominal obesity and circulating metabolites: a twin study approach," Metabolism: Clinical and Experimental, vol. 65, no. 3, pp. 111-121, 2016.

[26] P. K. Wiklund, S. Pekkala, R. Autio et al., "Serum metabolic profiles in overweight and obese women with and without metabolic syndrome," Diabetology and Metabolic Syndrome, vol. 6, article 40, 2014.

[27] J. Naukkarinen, S. Heinonen, A. Hakkarainen et al., "Characterising metabolically healthy obesity in weight-discordant monozygotic twins," Diabetologia, vol. 57, no. 1, pp. 167-176, 2014.

[28] P. Würtz, V.-P. Mäkinen, P. Soininen et al., "Metabolic signatures of insulin resistance in 7,098 young adults," Diabetes, vol. 61, no. 6, pp. 1372-1380, 2012.

[29] T. Chen, Y. Ni, X. Ma et al., "Branched-chain and aromatic amino acid profiles and diabetes risk in Chinese populations," Scientific Reports, vol. 6, article 20594, 2016.

[30] G. Xie, X. Ma, A. Zhao et al., "The metabolite profiles of the obese population are gender-dependent," Journal of Proteome Research, vol. 13, no. 9, pp. 4062-4073, 2014.

[31] L. Liu, R. Feng, F. Guo, Y. Li, J. Jiao, and C. Sun, "Targeted metabolomic analysis reveals the association between the postprandial change in palmitic acid, branched-chain amino acids and insulin resistance in young obese subjects," Diabetes Research and Clinical Practice, vol. 108, no. 1, pp. 84-93, 2015.

[32] M. M. Boulet, G. Chevrier, T. Grenier-Larouche et al., "Alterations of plasma metabolite profiles related to adipose tissue distribution and cardiometabolic risk," American Journal of Physiology_Endocrinology and Metabolism, vol. 309, no. 8, pp. E736-E746, 2016.

[33] F. Badoud, K. P. Lam, A. DiBattista et al., "Serum and adipose tissue amino acid homeostasis in the metabolically healthy obese," Journal of Proteome Research, vol. 13, no. 7, pp. 34553466, 2014.

[34] C. Takashina, I. Tsujino, T. Watanabe et al., "Associations among the plasma amino acid profile, obesity, and glucose metabolism in Japanese adults with normal glucose tolerance," Nutrition and Metabolism, vol. 13, article 5, 2016.

[35] C. Yamada, M. Kondo, N. Kishimoto et al., "Association between insulin resistance and plasma amino acid profile in non-diabetic Japanese subjects," Journal of Diabetes Investigation, vol. 6, no. 4, pp. 408-415, 2015. 
[36] T. Tillin, A. D. Hughes, Q. Wang et al., "Diabetes risk and amino acid profiles: cross-sectional and prospective analyses of ethnicity, amino acids and diabetes in a South Asian and European cohort from the SABRE (Southall And Brent REvisited) Study," Diabetologia, vol. 58, no. 5, pp. 968-979, 2015.

[37] J. Y. Kim, J. Y. Park, O. Y. Kim et al., "Metabolic profiling of plasma in overweight/obese and lean men using ultra performance liquid chromatography and Q-TOF Mass spectrometry (UPLC-Q-TOF MS)," Journal of Proteome Research, vol. 9, no. 9, pp. 4368-4375, 2010.

[38] E. S. Tai, M. L. S. Tan, R. D. Stevens et al., "Insulin resistance is associated with a metabolic profile of altered protein metabolism in Chinese and Asian-Indian men," Diabetologia, vol. 53, no. 4, pp. 757-767, 2010.

[39] A. E. Serralde-Zúñiga, M. Guevara-Cruz, A. R. Tovar et al., "Omental adipose tissue gene expression, gene variants, branched-chain amino acids, and their relationship with metabolic syndrome and insulin resistance in humans," Genes \& Nutrition, vol. 9, article 431, 2014.

[40] P. Whiting, A. W. S. Rutjes, J. B. Reitsma, P. M. M. Bossuyt, and J. Kleijnen, "The development of QUADAS: a tool for the quality assessment of studies of diagnostic accuracy included in systematic reviews," BMC Medical Research Methodology, vol. 3, pp. 1-13, 2003.

[41] L. C. Burrage, S. C. S. Nagamani, P. M. Campeau, and B. H. Lee, "Branched-chain amino acid metabolism: from rare Mendelian diseases to more common disorders," Human Molecular Genetics, vol. 23, no. 1, pp. R1-R8, 2014.

[42] J. Lu, G. Xie, W. Jia, and W. Jia, "Insulin resistance and the metabolism of branched-chain amino acids," Frontiers of Medicine in China, vol. 7, no. 1, pp. 53-59, 2013.

[43] K. C. Olson, G. Chen, Y. Xu, A. Hajnal, and C. J. Lynch, "Alloisoleucine differentiates the branched-chain aminoacidemia of Zucker and dietary obese rats," Obesity, vol. 22, no. 5, pp. 1212-1215, 2014.

[44] P. She, C. Van Horn, T. Reid, S. M. Hutson, R. N. Cooney, and C. J. Lynch, "Obesity-related elevations in plasma leucine are associated with alterations in enzymes involved in branched-chain amino acid metabolism," American Journal of PhysiologyEndocrinology and Metabolism, vol. 293, no. 6, pp. E1552-E1563, 2007.

[45] C. J. Lynch and S. H. Adams, "Branched-chain amino acids in metabolic signalling and insulin resistance," Nature Reviews Endocrinology, vol. 10, no. 12, pp. 723-736, 2014.

[46] S. H. Um, F. Frigerio, M. Watanabe et al., "Absence of S6K1 protects against age- and diet-induced obesity while enhancing insulin sensitivity," Nature, vol. 431, pp. 200-205, 2004.

[47] M. E. Patti, E. Brambilla, L. Luzi, E. J. Landaker, and C. R. Kahn, "Bidirectional modulation of insulin action by amino acids," The Journal of Clinical Investigation, vol. 101, no. 7, pp. 1519-1529, 1998.

[48] T. M. Pederson, D. L. Kramer, and C. M. Rondinone, "Serine/threonine phosphorylation of IRS-1 triggers its degradation: possible regulation by tyrosine phosphorylation," Diabetes, vol. 50, no. 1, pp. 24-31, 2001.

[49] M. Doi, I. Yamaoka, M. Nakayama, K. Sugahara, and F. Yoshizawa, "Hypoglycemic effect of isoleucine involves increased muscle glucose uptake and whole body glucose oxidation and decreased hepatic gluconeogenesis," American Journal of Physiology -Endocrinology and Metabolism, vol. 292, no. 6, pp. E1683-E1693, 2007.
[50] J. A. B. Pedroso, T. T. Zampieri, and J. Donato, "Reviewing the effects of l-leucine supplementation in the regulation of food intake, energy balance, and glucose homeostasis," Nutrients, vol. 7, no. 5, pp. 3914-3937, 2015.

[51] P. Felig, E. Marliss, and G. F. Cahill Jr., "Plasma amino acid levels and insulin secretion in obesity," The New England Journal of Medicine, vol. 281, no. 15, pp. 811-816, 1969.

[52] K. H. Pietiläinen, J. Naukkarinen, A. Rissanen et al., "Global transcript profiles of fat in monozygotic twins discordant for BMI: pathways behind acquired obesity," PLoS Medicine, vol. 5, no. 3, pp. 472-483, 2008.

[53] A. Solini, E. Bonora, R. Bonadonna, P. Castellino, and R. A. Defronzo, "Protein metabolism in human obesity: relationship with glucose and lipid metabolism and with visceral adipose tissue," Journal of Clinical Endocrinology \& Metabolism, vol. 82, no. 8, pp. 2552-2558, 1997.

[54] M. A. Herman, P. She, O. D. Peroni, C. J. Lynch, and B. B. Kahn, "Adipose tissue Branched Chain Amino Acid (BCAA) metabolism modulates circulating BCAA levels," The Journal of Biological Chemistry, vol. 285, no. 15, pp. 11348-11356, 2010.

[55] A. C. Shin, M. Fasshauer, N. Filatova et al., "Brain insulin lowers circulating bcaa levels by inducing hepatic bcaa catabolism," Cell Metabolism, vol. 20, no. 5, pp. 898-909, 2014.

[56] S. Chevalier, S. C. Burgess, C. R. Malloy, R. Gougeon, E. B. Marliss, and J. A. Morais, "The greater contribution of gluconeogenesis to glucose production in obesity is related to increased whole-body protein catabolism," Diabetes, vol. 55, no. 3, pp. 675-681, 2006.

[57] M. Krebs, M. Krssak, E. Bernroider et al., "Mechanism of amino acid-induced skeletal muscle insulin resistance in humans," Diabetes, vol. 51, no. 3, pp. 599-605, 2002.

[58] A.-M. Davila, F. Blachier, M. Gotteland et al., "Re-print of 'intestinal luminal nitrogen metabolism: role of the gut microbiota and consequences for the host"' Pharmacological Research, vol. 69, no. 1, pp. 114-126, 2013.

[59] Human Microbiome Project Consortium, "Structure, function and diversity of the healthy human microbiome," Nature, vol. 486, no. 7402, pp. 207-214, 2012.

[60] Z.-L. Dai, G. Wu, and W.-Y. Zhu, "Amino acid metabolism in intestinal bacteria: links between gut ecology and host health," Frontiers in Bioscience, vol. 16, no. 5, pp. 1768-1786, 2011.

[61] B. A. Irving, R. E. Carter, M. Soop et al., "Effect of insulin sensitizer therapy on amino acids and their metabolites," Metabolism: Clinical and Experimental, vol. 64, no. 6, pp. 720-728, 2015.

[62] G. A. Walford, J. Davis, A. S. Warner et al., "Branched chain and aromatic amino acids change acutely following two medical therapies for type 2 diabetes mellitus," Metabolism: Clinical and Experimental, vol. 62, no. 12, pp. 1772-1778, 2013. 


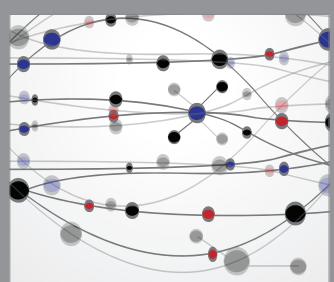

The Scientific World Journal
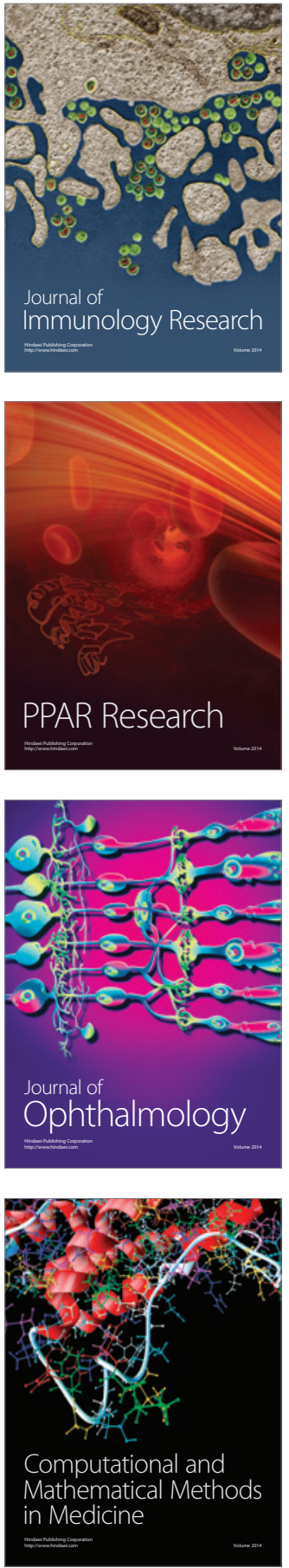

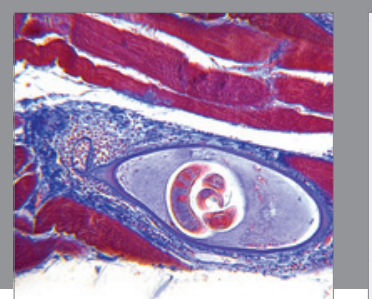

Gastroenterology Research and Practice

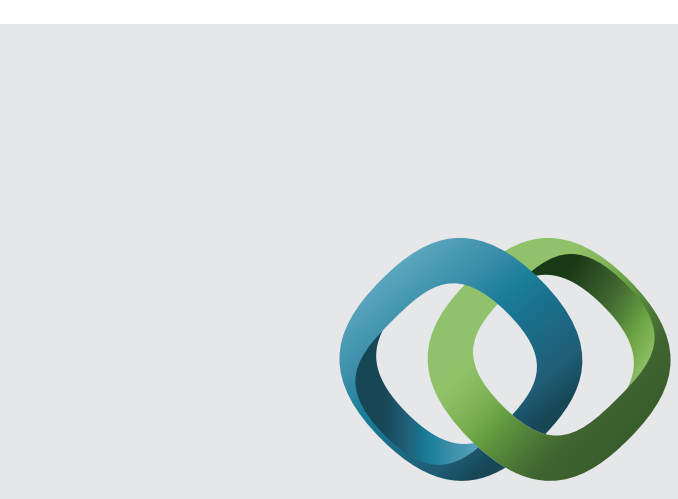

\section{Hindawi}

Submit your manuscripts at

http://www.hindawi.com
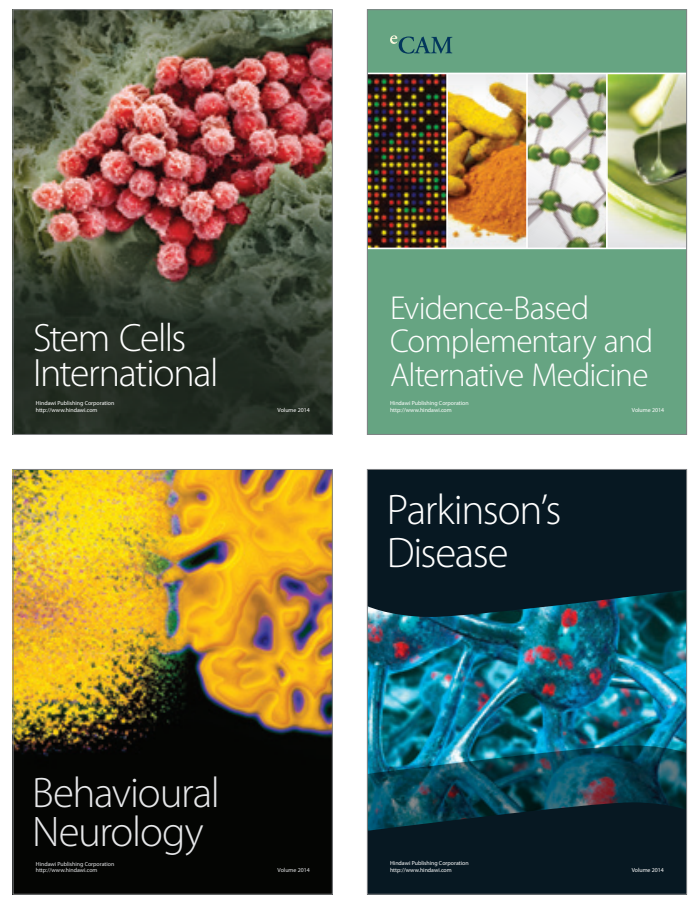
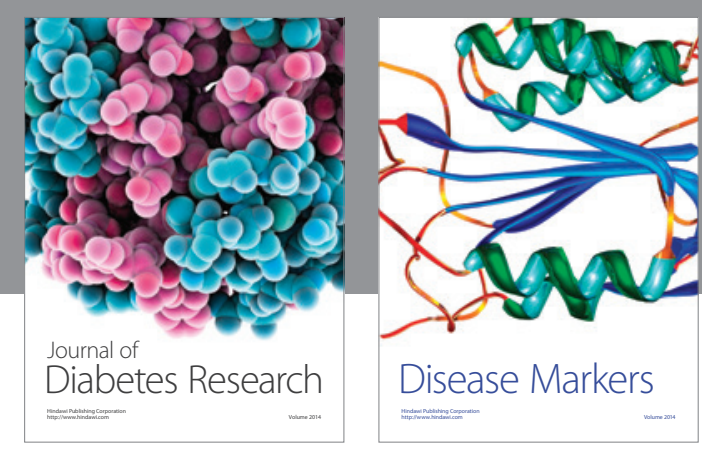

Disease Markers
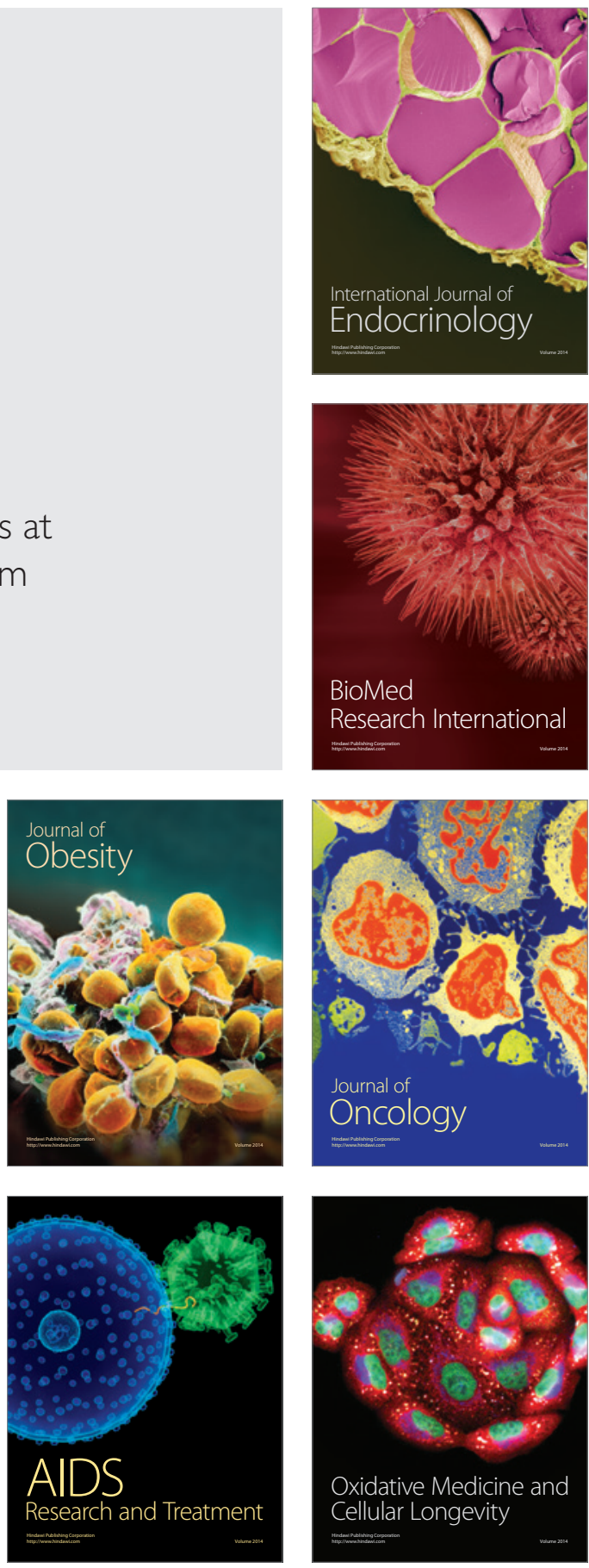